\title{
Endoscopic surgical skill qualification and educational system of the Japanese Orthopaedic Association
}

\author{
Akira Dezawa \\ Department of Orthopedic Surgery, Teikyo University Mizonokuchi Hospital, Tokyo 173-8605, Japan. \\ Correspondence to: Dr. Akira Dezawa, Department of Orthopedic Surgery, Teikyo University Mizonokuchi Hospital, Tokyo 173-8605, Japan. \\ E-mail: adezawajp@gmail.com
}

How to cite this article: Dezawa A. Endoscopic surgical skill qualification and educational system of the Japanese Orthopaedic Association. Miniinvasive Surg 2017;1:52-5.

\author{
Article history: Received: 17-04-2017 Accepted: 07-05-2017 Published: 30-06-2017
}

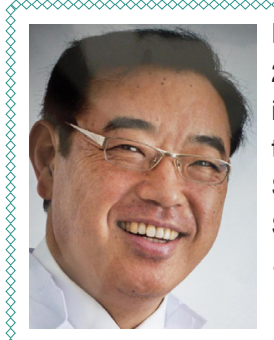

Dr. Akira Dezawa, M.D., Ph.D, is Deputy Director at Teikyo University School of Medicine Mizonokuchi Hospital since 2006. He also services as the Professor in Department of Orthopedics at Teikyo University since 2004. His expertise includes endoscopic spinal surgery and he is a well-known physician for the minimally invasive spine surgery. He is the 2nd President of The Pacific Asian Society of the Minimally Invasive Spine Surgeries, the 1st President of Japan Society of the Study of Spinal Endoscopy, the 7th President of WCMISST (2020), the 1st President of Japan PED Society, Japanese Represent of ISMISS, Editorial Board of European Spine Journal, Asian Journal of Endoscopic Surgery, Asian Spine Journal.

\section{INTRODUCTION}

In the field of spinal surgery, the application of minimally invasive endoscopic surgery owes much to advances in high performance optical instruments [chargecoupled device cameras and the latest small diameter (just $2 \mathrm{~mm}$ ) endoscopes and electronic scopes] and the accompanying improvements in imaging technology and the development of innovations such as ultrasonic incision devices. As far as the manipulation for decompression of the spinal nerve root is concerned, reliability and safety both improved following development and advances in instruments such as robotics and enhanced endoscopic resolution, and further improvement is anticipated henceforth. In Japan, endoscopy began to be applied in 1995 to anterior approach spinal surgery and in 1997 to posterior approach surgery. After this form of surgery began to be covered by the national health insurance, endoscopic spinal surgery spread rapidly. However, as this form of surgery spread rapidly, complications arising from the procedures began to gradually be highlighted as a problem. The steps to establish an endoscopic surgical skill qualification qualification (ESSQ) system were started in 2002 and this system was launched at the Japanese Orthopaedic Association (JOA) in 2004..$^{[1]}$ This system, called the "endoscopic spinal surgery skill qualification system", is aimed at "evaluating the skills of individual surgeon involved in endoscopic spinal surgery in accordance with common criteria and accrediting surgeons satisfying certain highlevel standards", with the ultimate goal of facilitating

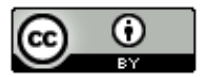

This is an open access article licensed under the terms of Creative Commons Attribution 4.0 International License (https://creativecommons.org/licenses/by/4.0/), which permits unrestricted use, distribution, and reproduction in any medium, as long as the original author is credited and the new creations are licensed under the identical terms.

For reprints contact: service@oaepublish.com
}

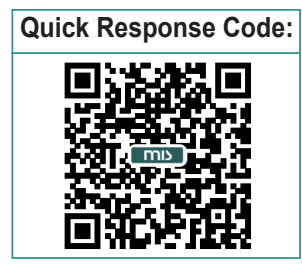


the sound spread and progress of endoscopic spinal surgery in Japan. ${ }^{[2]}$

\section{COURSE AND METHODS OF QUALIFICATION AND RESULTS}

Qualification procedures for skills are as follows. First, the applicant, satisfying the requirements for filing an application for skill qualification, submits the necessary documents and an unedited videotape. On the basis of the submitted materials, the skill of each application is assessed by multiple referees (referee committee on surgical skill qualification). Assessments are based on scoring of each checked item (full score: 100 per item).

During assessment of the videotape, if one referee raises an objection, another referee or all members of the referee committee conduct further evaluations. If two or more referees raise objections, the applicant is automatically judged to be "unacceptable" for qualification. The referee qualification must be updated every fifth year. Each referee applying for renewal of the referee qualification has several obligations, including submission of a certificate of clinical activity for 5 consecutive years.

The first application from surgeons for skill qualification under this system was received in December 2004. Through strict assessment of the documents, DVD and videotape submitted, on April 1, 2005, 6 surgeons were accredited as to the anterior approach skill and 18 surgeons as to the posterior approach. ${ }^{[2]}$

We sent a questionnaire to facilities registered with JOA in advance as spine surgery special hospital. During the period from 2005 to 2014, responses to the questionnaire were collected from 1,148 facilities per year on average. The mean response rate was $56.4 \%$ (45.2-63.9\%).

The number of facilities providing endoscopic surgery averaged 262.9 (208-297), with the mean percentage being $24.8 \%(20.3-27.3 \%)$.

Under this qualification system, 167 surgeons were qualified in terms of endoscopic surgical skill between 2004 and 2015. The posterior approach skill (MED, PELD) qualification was obtained by 165 surgeons (MED: 141, PELD: 24) and the anterior approach skill qualification by 2 surgeons [Table 1].

The mean percentage of applicants who qualified successfully was $50.6 \%(39.1-80 \%)$ after video assessments. Following the recent increase in endoscopic surgery, the number of complications arising from this form of surgery has tended to rise annually (from 66 cases in 2005 to 361 cases in 2014). However, the mean incidence of complications per year has remained essentially unchanged at $2.4 \%$ $(1.57-2.81 \%)$ [Table 1]..$^{[3,4]}$

\section{PRINCIPLE AND SIGNIFICANCE OF ENDOSCOPIC SPINAL SURGERY SKILL QUALIFICATION SYSTEM}

It loses trust of the whole endoscopic surgery that complications of the endoscopic surgery in the field of spine increase, serving as a major obstacle slowing and even blocking advances in endoscopic surgery at all related specialties. This is a serious problem for all surgeons involved in endoscopic surgery. Every time such complications arise, citizens, the mass media and governmental organs begin arguing about the necessity of establishing an educational system and a qualification assessment organization/system for endoscopic surgeons. The JOA rules on the skill qualification system were drafted taking this viewpoint into account. The rules are based on two principles: (1) the system is aimed at accrediting endoscopic spinal surgeons with sufficient skills through assessment in accordance with high-level standards; and (2) the system is aimed at evaluating the skill level of only surgeons engaged in endoscopic spinal surgery and is not based on the specialist surgeon system set forth by the specialist surgeon accreditation council. The significance of establishing this qualification system lies in: (1) the endoscopic spinal surgeon's skill qualification in accordance with high-level standards makes endoscopic spinal surgeons more acceptable to the public and raises their value; (2) an increase in the number of qualified surgeons and their activities will operate favorably as a mechanism to mitigate

Table1: Changes over time from 2004 to 2015 in the number of applicants for skill qualification and the percentage of successfully qualified applicants

\begin{tabular}{lccc}
\hline Year & Board certified surgeon & Applicant & Success ratio (\%) \\
\hline 2004 & 24 & 30 & 80 \\
2005 & 8 & 14 & 53 \\
2006 & 14 & 30 & 45 \\
2007 & 17 & 31 & 55 \\
2008 & 14 & 31 & 45 \\
2009 & 13 & 32 & 41 \\
2010 & 13 & 26 & 50 \\
2011 & 15 & 30 & 50 \\
2012 & 14 & 30 & 47 \\
2013 & 20 & 35 & 57 \\
2014 & 16 & 27 & 59 \\
2015 & 15 & 32 & 47 \\
\hline
\end{tabular}


risk, contributing to the sound spread and progress of endoscopic spinal surgery in Japan; and so on.$^{[3]}$

\section{ACTIONS RELATED TO THE SKILL QUALIFICATION SYSTEM}

\section{Guidelines on endoscopic spinal surgery}

1. Before endoscopic spinal surgery, preparatory steps need to be taken in accordance with the rules prevailing at the facility concerned.

2. Thoracoscopic surgery should be performed under the supervision of a surgeon qualified by JOA to perform endoscopic spinal surgery, who has acquired sufficient skills in open chest surgery and can cope with any complications arising during or after surgery appropriately (hereinafter called "skill-qualified surgeon").

3. Laparoscopic or posterior laparoscopic surgery should be performed under the supervision of a skillqualified surgeon who has acquired sufficient skills in open abdominal surgery and the retroperitoneal approach and can cope with any complications arising during or after surgery appropriately. ${ }^{[5]}$

4. If thoracotomy or laparotomy is required during endoscopic surgery, the surgery should be immediately switched to open chest or open abdominal surgery. It must be ensured that cooperation from a thoracic surgeon or an abdominal surgeon is always available, in the event of being needed. ${ }^{[6]}$

5. Preoperative and postoperative patient management is conducted under a system in which the surgeon plays a central role.

\section{Requirements before endoscopic spinal surgery.}

(1) Learning open chest/abdominal surgery procedure and perioperative management and how to deal with complications;

(2) Understanding the anatomical structure and relative position of each organ during endoscopy;

(3) Mastering the approaches with a thoracoscope, laparoscope and posterior/posterolateral spinal endoscope;

(4) Mastering the sense of depth under two-dimensional video monitor images;

(5) Mastering the sense of organ touch by remote control;

(6) Mastering coordination between visual sense and finger motions under magnified images;

(7) Mastering how to use special tools/devices;

(8) Mastering the special skills required for endoscopic surgery (ligating method, etc.).

Of those listed above, 2 through 8 should be studied and fully mastered by attending the endoscopic spinal surgery education/training courses provided or authorized by the JOA.

7. Requirement of surgeons performing anterior approach endoscopic spinal surgery: having experienced the anterior approach spinal surgery in at least 20 cases.

8. Requirement of surgeons performing posterior/ posterolateral approach endoscopic spinal surgery: having experienced the posterior/posterolateral approach spinal surgery in at least 30 cases.

9. Informed consent, based on a decision made by the patient after sufficient explanation, must be obtained before endoscopic spinal surgery.

10. In the event of a near-miss or an actual accident during endoscopic spinal surgery, primary emphasis needs to be placed on securing of the patient's safety and appropriate actions must be taken promptly in accordance with all relevant hospital rules. At the same time, an endoscopic spinal surgery near-miss/accident report needs to be submitted to the "Endoscopic Spinal Surgery Skill Qualification Committee" (c/o JOA Secretariat) ${ }^{[6]}$

\section{Educational system and maintenance of qualified skills}

Training methods can be roughly divided into training with the use of animals (pigs, sheep), participation in training courses, supervision by endoscopic surgeons, implementation of existing open chest/ abdominal surgery under endoscopic guidance, and so on. Japanese Society for the Study of Endoscopic

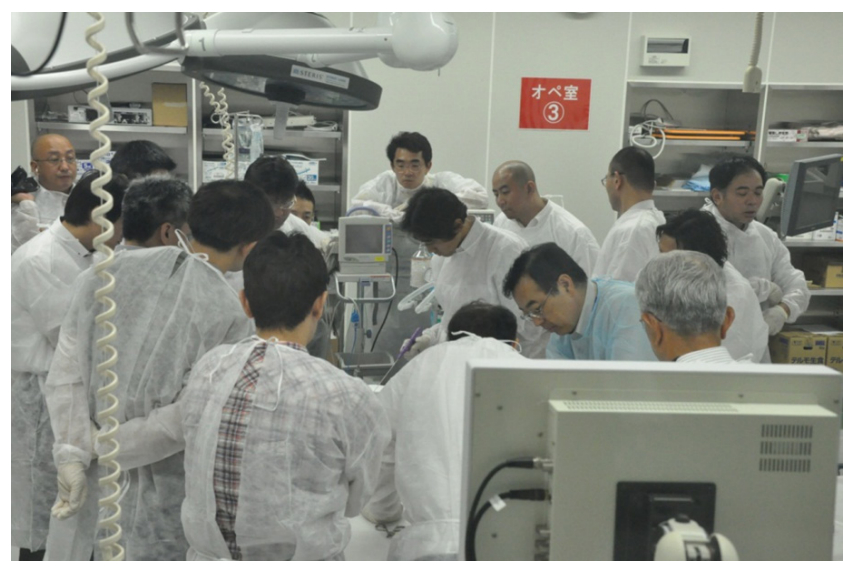

Figure 1: Animal surgery allows the surgeon to practice important skills needed during actual surgery 
and Minimally Invasive Spine Surgery (JESMISS) established in 1999 and changed the name with Japanese Society of Minimally Invasive Spine Surgery (JASMISS) in 2015. JESMISS has been making efforts to implement training with the use of pigs for coping with the qualification system. In the field of orthopedic surgery, 5 in vitro training sessions on spinal endoscopy were officially provided, beginning with the 14th Arthroscopy Seminar in July 1996, and an in vivo training session was provided during the First Endoscopic Spinal Surgery Seminar in September 1997. To date, 14 JASMISS training sessions and 13 JOA training sessions have been provided, involving a total of 1,020 participants [Figure 1].

\section{CONCLUSION}

This system is designed to provide accreditation of sufficient skills to surgeons for the purpose of facilitating endoscopic spinal surgery progress in an appropriate direction. We believe that this system will ensure the provision of safe and reliable endoscopic spinal surgery.

\section{Authors'contributions}

A. Dezawa contributed solely to this paper.

\section{Financial support and sponsorship}

None.

\section{Conflicts of interest}

There are no conflicts of interest.

\section{Patient consent}

Written informed consent was obtained from the patient.

\section{Ethics approval}

This article is approved by the Ethical Committee of Teikyo University Mizonokuchi Hospital.

\section{REFERENCES}

1. Dezawa A. Current status of surgical skill qualification system. J Jan Endosc Surg 2003;8:115-21.

2. Dezawa A. Spine endoscopic surgical skill qualification system of Japanese Orthopedic Association. In: Dezawa A, Shinomiya K, editors. Endoscopic Spine Surgery. Tokyo: Nankodo; 2007. p. 6-12.

3. Konno S. Current status of endoscopic spine surgery-incident cases of annual report 2014-. J Jpn Orthop Assoc 2016;90:41-7.

4. Matsumoto M. Current status of endoscopic spine surgery-incident cases of annual report 2007-. J Jpn Orthop Assoc 2009;83:56-61.

5. Matsuda T, Ono Y, Terachi T, Naito S, Baba S, Miki T, Hirao Y, Okuyama A. The endoscopic surgical skill qualification system in urological laparoscopy: a novel system in Japan. $J$ Urol 2006;176:2168-72.

6. Tanigawa N, Lee SW, Kimura T, Mori T, Uyama I, Nomura E, Okuda J, Konishi F. The Endoscopic Surgical Skill Qualification System for gastricsurgeryin Japan. Asian J Endosc Surg 2011;4:112-5. 